\title{
Níveis pressóricos de pacientes em acompanhamento pelo Programa Hiperdia
}

\author{
Pressure levels of patients followed by the Hiperdia Program
}

Eduardo Tavares Gomes ${ }^{1}$, Simone Maria Muniz da Silva Bezerra`

${ }^{1}$ Programa Associado de Pós-Graduação em Enfermagem, Universidade de Pernambuco e Universidade Estadual da Paraíba (UPE/UEPB) - Recife (PE), Brasil

DOI: http://dx.doi.org/10.7322/abcshs.v43i2.1076

\section{RESUMO}

Introdução: O Programa Hiperdia é uma ação importante para a prevenção das doenças crônicas não-transmissíveis no Brasil, particularmente no controle da hipertensão arterial e diabetes. Objetivo: Comparar os níveis pressóricos de pacientes hipertensos em acompanhamento pelo Programa Hiperdia da Estratégia de Saúde da Família do munícipio de Recife-PE, em relação a uma amostra da população local sem acompanhamento. Métodos: Estudo transversal, analítico, de abordagem quantitativa, realizado com um grupo de 135 pacientes hipertensos do Programa Hiperdia e 196 pacientes sem vínculo com uma unidade de saúde ou sem diagnóstico prévio de hipertensão arterial como grupo controle. Resultados: Os pacientes do programa Hiperdia apresentaram-se como controlados (73,3\%) e com médias de pressão arterial diastólica e sistólica menor que o grupo controle ( $p<0,001$ e $p=0,011$, respectivamente). Conclusão: Os resultados ressaltam a importância do programa e a necessidade de estratégias para melhorar o diagnóstico na população em geral.

Palavras-chave: estratégia saúde da família; hipertensão; saúde pública; equipe de enfermagem.

\section{ABSTRACT}

Introduction: The Hiperdia Program is an important action for the prevention of chronic non-communicable diseases in Brazil, particularly in the control of hypertension and diabetes. Objective: To compare the blood pressure of hypertensive patients from Hiperdia Program of the Family Health Strategy from Recife-PE, in relation to a sample of the local population not enrolled in a control program. Methods: Cross-sectional, analytical study with a quantitative approach, conducted with a group of 135 hypertensive patients from the Hiperdia program and 196 patients without obligatory link to a health service or without previous diagnosis of hypertension as a control group. Results: Patients enrolled in the Hiperdia program were controlled (73.3\%) and mean diastolic and systolic blood pressure were lower than control group ( $p<0.001$ and $p=0.011$, respectively). Conclusion: The results point to the importance of the program and the need for strategies to improve diagnosis in the general population.

Keywords: family health strategy; hypertension; public health; nursing, team. 


\section{INTRODUÇÃO}

A Hipertensão Arterial Sistêmica (HAS) é o principal fator de risco para as doenças cardiovasculares. Essa condição de saúde está associada à importante morbimortalidade e incapacidade física, além de altos custos ${ }^{1}$. É uma condição crônica que já responde um dos principais problemas de saúde nos países desenvolvidos, devido a sua alta prevalencia e o seu papel reconhecido como um factor de risco cardiovascular ${ }^{2-4}$.

A HAS é uma doença com impacto financeiro significativo para os pacientes, familiares e para sociedade, devido a sua natureza crônica, suas complicações e os meios utilizados para o seu controle e tratamento, além de custos intangíveis (efeitos colaterais, ansiedade, transtorno e perda da qualidade de vida $)^{5}$.

Trata-se de um problema de saúde crônico que, após o diagnóstico, acarretará em visitas regulares de controle, modificações de estilo de vida e início de tratamento farmacológico em situações específicas para tentar impedir o desenvolvimento de complicações ${ }^{6}$.

O incentivo a medidas terapêuticas não-farmacológicas deve implicar em promoção da saúde por melhorar a compreensão da doença e seus efeitos, estimular hábitos saudáveis, guiar uma mudança no estilo de vida e no uso de medicamentos e seus efeitos adversos, bem como por incentivar à participação do paciente em programas de autocuidado ${ }^{7,8}$.

Para a ampliação do espaço de educação, orientação e acompanhamento dos pacientes hipertensos na Estratégia de Saúde da Família, o Hiperdia foi criado por meio Portaria n 371/GM, em 4 de março de 2002, mediante um Plano de Reorganização da Atenção à Hipertensão Arterial e Diabetes Mellitus ${ }^{9}$. Através de reuniões, geralmente mensais e sob coordenação do enfermeiro, a equipe mantém a orientação permanente sobre a doença e os pacientes encontram espaço para compartilhar suas experiências, dificuldades.

As equipes de saúde devem, ao cuidar do paciente hipertenso, ter por meta principal garantir a adesão do indivíduo ao tratamento ${ }^{10}$. Estudos que avaliem o controle da pressão arterial tornam-se ferramentas imprescindíveis ao trabalho do profissional de saúde que atua nessa área.

Através desse estudo objetivou-se comparar os níveis pressóricos de pacientes hipertensos em acompanhamento pelo Programa Hiperdia da Estratégia de Saúde da Família do munícipio de Recife-PE, em relação a uma amostra da população local sem acompanhamento.

\section{MÉTODOS}

Estudo transversal, analítico, de abordagem quantitativa, realizado com um grupo de 135 pacientes hipertensos acompanhados pelo Programa Hiperdia de uma Unidade de Saúde da Família do Distrito Sanitário II do município de Recife-PE. Para grupo controle, foram incluídos 196 pacientes sem obrigatoriedade do vínculo com uma unidade de saúde ou diagnóstico prévio de hipertensão arterial. A amostra foi censitária, com estimativa inicial de 196 pacientes, número de acompanhados pela unidade. Para o controle, foi considerado um individuo para cada paciente. Contudo, a amostra em acompanhamento no Hiperdia ficou menor que o estimado devido ao absenteísmo dos pacientes no período da coleta.

A coleta de dados do grupo de pacientes acompanhados pelo Hiperdia ocorreu nos meses de novembro a dezembro de 2016, nas reuniões do grupo na própria unidade ou em locais da comunidade. Para o grupo controle, os pacientes foram abordados em ações educativas extensionistas em locais públicos fora da área do distrito sanitário II, diminuindo a possibilidade de serem abordados os mesmos pacientes nos dois grupos. Os grupos não tinham membros em comum. A elaboração do grupo controle foi norteada pelo intuito de ter-se uma amostra da população geral, sem acompanhamento pelo programa para análise comparativa, ou seja, não foram incluídos pacientes que participassem de grupos de Hiperdia em unidades de saúde do distrito ou de quaisquer outras, podendo participar pacientes com ou sem diagnóstico prévio de hipertensão arterial. Dessa forma, objetivou-se construir um grupo controle em que os participantes soubessem ou não da hipertensão, mas que não fossem alvo de estratégias de educação em saúde para prevenção primária e secundária, como para promover a adesão farmacológica ou não.

Os pacientes eram convidados a participarem das entrevistas e da avaliação física. Na coleta que compôs o grupo controle, a atividade educativa levou à comunidade presente nos locais palestras, panfletos e orientações sobre hipertensão arterial, com conteúdo similar ao utilizado rotineiramente no grupo Hiperdia. A população recebeu o mesmo serviço, mesmo para os que por qualquer motivo não desejaram participar da amostra. Não participaram da amostra pacientes menores de 18 anos, em ambos os grupos.

Foram analisados dados sociodemográficos (idade, sexo, cor da pele, anos de estudos e situação conjugal) e clínicos (pressão arterial sistólica e diastólica, tempo de diagnóstico de HAS, Índice de Massa Corporal (IMC), co-morbidades e características clínicas do paciente hipertenso). Nos casos de dúvida os dados eram confirmados no prontuário de cadastro estabelecido pelo Hiperdia. A medida do peso na faixa ideal foi obtida pelo IMC, calculado a partir dos valores de peso e altura e classificado de acordo com a Organização Mundial de Saúde em peso normal $\left(\geq 18,5 \mathrm{Kg} / \mathrm{m}^{2}\right.$ e $\left.<25 \mathrm{Kg} / \mathrm{m}^{2}\right)$, sobrepeso $\left(\geq 25 \mathrm{~kg} / \mathrm{m}^{2}\right.$ e $\left.<30 \mathrm{~kg} / \mathrm{m}^{2}\right)$ e obesidade $\left(\geq 30 \mathrm{Kg} / \mathrm{m}^{2}\right)^{5}$.

A pressão arterial (PA) foi medidautilizando-se o aparelho automático digital validado e foram seguidas as recomendações para aferição da PA da VI Diretrizes Brasileiras de Hipertensão ${ }^{5}$. Para a realização de análises, os valores da PA foram estratificados conforme os estágios de HAS, seguindo as diretrizes brasileiras de hipertensão, em controlados ( $\mathrm{PA}<140 / 90 \mathrm{mmHg}$ ), hipertensão estágio 1 (PAS entre 140-159 e PAD entre 90-99); estágio 2 (PAS entre 160-179 e PAD entre 100-109) e estágio 3 $(\mathrm{PAS}>180 \text { e } \mathrm{PAD}>110)^{5}$. 
Os dados obtidos foram transcritos para meio eletrônico com auxílio do programa estatístico Epi-info7.0. Após a entrada dos dados, os mesmos foram revisados para evitar inconsistências por digitação, não havendo nenhum paciente coletado sido desprezado da amostra por falha na coleta. Através do teste de Normalidade Kolmogorov-Smirnov foram verificados que os desfechos de pressão sistólica e diastólica avaliados apresentaram distribuição normal $(\mathrm{p}<0,05)$. A análise dos dados consistiu na estatística descritiva das variáveis (frequências absolutas e relativas, média, desvio padrão), o teste não-paramétrico de MannWhitney para comparação das médias das variáveis numéricas de forma dicotômica. $\mathrm{O}$ nível de significância aceito para os testes estatísticos foi de 0,05 (ou 5\%).

O projeto de pesquisa foi submetido e aprovado pelo Comitê de Ética em Pesquisa do Complexo Hospitalar HUOC/PROCAPE (CAAE 01650112.8.0000.5192; Parecer $\mathrm{n}^{\circ}$ 121.849). Os sujeitos foram convidados, esclarecidos e registraram o consentimento em participar através do Termo de Consentimento Livre e Esclarecido. Independente de participarem ou não da amostra, os convidados tiveram a oportunidade de serem avaliados e esclarecidos quanto aos seus resultados, bem como participar das atividades educativas oferecidas. A composição do grupo controle também serviu de rastreamento precoce na comunidade e de alerta para muitos dos que participaram que desconheciam a doença e receberam esclarecimentos e orientações quanto à busca pelo serviço de saúde.

\section{RESULTADOS}

A amostra dos pacientes que são acompanhados pela unidade mostrou-se com média de idade de 49,88 $\pm 17,79$ anos e apenas $28,89 \%$ maiores de 60 anos e predominância de mulheres $(68,1 \%)$. O peso médio foi de $72,08 \pm 18,24 \mathrm{~kg}$, sendo a maior parte dos pacientes na faixa acima do peso normal, ou seja, em sobrepeso $(42,2 \%)$ e obesidade $(18,5 \%)$ e índice de massa corporal médio de $26,66 \pm 5,27 \mathrm{~kg} / \mathrm{m}^{2}$ (Tabela 1 ).

Os níveis pressóricos encontrados neste grupo revelam bom controle, com PAS média de 128,0 $\pm 15,78 \mathrm{mmHg}$, PAD média de $81,33 \pm 10,49 \mathrm{mmHg}$, sendo a maioria classificada como na faixa normal $(45,2 \%)$ e limítrofe $(28,1 \%)$, apenas $26,7 \%$ com níveis considerados hipertensivos e nenhum paciente em estágio 3 (Tabela 1).

Quanto aos hábitos de vida relacionados à saúde, 85,2\% referiu-se como não-fumante, sendo 5,9\% ex-fumante e apenas $8,9 \% \mathrm{fu}$ mante. $\mathrm{O}$ etilismo esteve presente em foi apresentado por mais entrevistados, correspondendo a $35,6 \%$ da amostra. Apenas $4,4 \%$ relataram uso de álcool maior que três vezes semanais de frequência. A atividade física regular foi relatada por $37,5 \%$ dos participantes, sendo $25,18 \%$ em até três vezes semanais (Tabela 1 ).

O grupo Hiperdia mostrou-se significativamente mais jovem que o controle $(\mathrm{p}=0,008)$, contudo a média de idade de ambos ficou abaixo de 60 anos. $\mathrm{O}$ sexo feminino foi predominante em ambos os grupos, sendo menor a diferença entre a frequência dos gêneros no grupo controle $(\mathrm{p}=0,008)$. A média de peso do grupo acompanhado foi significativamente mais baixo $(p=0,014)$ que a média do grupo controle $(74,36 \pm 13,37 \mathrm{~kg})$. O mesmo ocorreu entre o IMC $(\mathrm{p}<0,001)$, estando ambos os grupos na faixa do sobrepeso e o grupo controle mais próximo à obesidade. O sobrepeso e a obesidade tiveram frequências significativamente $(\mathrm{p}<0,001)$ maiores no grupo controle $(80,1 \%)$. Os níveis pressóricos do grupo controle apresentaram-se também significativamente maiores, podendo considerar-se o grupo não-controlado, com média na faixa do primeiro estágio da hipertensão (PAS=143,06 $\pm 22,8 \mathrm{mmHg}, \mathrm{p}<0,001 ; \mathrm{PAD}=84,07 \pm 15,55 \mathrm{mmHg}$, $\mathrm{p}=0,011)$ (Tabela 2).

\section{DISCUSSÃO}

O perfil dos pacientes acompanhados pelo programa Hiperdia revelou-se similar a outros estudos com essa população em outras localidades, apresentando a predominância de mulheres, mas apresentou-se mais jovem ${ }^{1,3,10}$. A elevada prevalência de sobrepeso e obesidade também foi relatada em outras pesquisas ${ }^{4,11}$. No entanto, apesar da elevada prevalência de pacientes nessas faixas, o peso e o índice de massa corporal foram significativamente menores que a amostra da população.

Tabela 1: Caracterização da amostra em acompanhamento pelo Programa Hiperdia. ( $\mathrm{N}=135)$ Recife-PE, 2016

\begin{tabular}{|l|c|}
\hline Variáveis & $\begin{array}{c}\text { N }(\%) \text { ou } \\
\text { Média } \pm \text { Desvio-Padrão }\end{array}$ \\
\hline Idade (anos) & $49,88 \pm 17,79$ \\
\hline$<60$ anos & $96(71,11)$ \\
\hline$>60$ anos & $39(28,89)$ \\
\hline Homens & $43(31,9)$ \\
\hline Mulheres & $92(68,1)$ \\
\hline Peso $(\mathrm{kg})$ & $72,08 \pm 18,24$ \\
\hline Peso normal & $53(39,3)$ \\
\hline Sobrepeso & $57(42,2)$ \\
\hline Obesidade & $25(18,5)$ \\
\hline Altura (m) & $1,64 \pm 0,09$ \\
\hline IMC (kg/m²) & $26,66 \pm 5,27$ \\
\hline PAS (mmHg) & $128,0 \pm 15,78$ \\
\hline PAD (mmHg) & $81,33 \pm 10,49$ \\
\hline PA Controlada & $99(73,3)$ \\
\hline HAS 1 & $31(23)$ \\
\hline HAS 2 & $5(3,7)$ \\
\hline Não-fumante & $115(85,2)$ \\
\hline Ex-fumante & $8(5,9)$ \\
\hline Fumantes & $12(8,9)$ \\
\hline Etilismo & $48(35,6)$ \\
\hline Até 3x/ semana & $42(31,1)$ \\
\hline Mais de 3x/semana & $6(4,4)$ \\
\hline Atividade Física & $50(37,5)$ \\
\hline Até 3x/ semana & $34(25,18)$ \\
\hline Mais de 3x/semana & $16(11,85)$ \\
\hline \hline & \\
\hline
\end{tabular}

$\mathrm{IMC}=$ índice de massa corporal; $\mathrm{PA}=$ pressão arterial; $\mathrm{PAS}=$ pressão arterial sistólica; PAD = pressão arterial diastólica; HAS $1=$ Hipertensão Arterial estágio 1; HAS 2 = Hipertensão Arterial estágio 2. 
Os níveis pressóricos encontrados retratam níveis controlados, com médias similares a de outros estudos avaliando o programa Hiperdia ${ }^{1}$. O percentual de pacientes considerados controlados varia entre os estudos nacionais, chegando até a $91 \%{ }^{10}$. Um estudo recente realizado com 330 pacientes em acompanhamento pelo programa na cidade de Vitória/ES apresentou níveis pressóricos algo acima dos achados apresentados, correlacionando de forma significativa o aumento da pressão arterial ao risco coronariano ${ }^{12}$.

Em um estudo realizado em Maceió/AL, o controle pressórico foi associado a três pontos importantes no acompanhamento dos hipertensos: menor número de medicamentos, menor queixa de efeitos colaterais e maior tempo de tratamento ${ }^{10}$. Para esse estudo, os principais efeitos colaterais relatados foram: ressecamento de mucosa, tosse seca, gosto metálico, cefaleia, náuseas ${ }^{10}$. O controle pressórico de $91 \%$ encontrado nessa pesquisa ocorre paradoxalmente a adesão dos pacientes as ações do próprio programa, avaliada em $40 \%^{10}$

Dos resultados apresentados, chama particular atenção a média pressórica no grupo controle, caracterizando-o como em hipertensão. Esse achado reforça a necessidade de campanhas educativas e ações de promoção de saúde como a realizada visto que grande parte da população apresenta a hipertensão de forma silenciosa, permanecendo não-tratada, enquanto ao programa Hiperdia se mostra como alternativa gratuita e eficaz para o controle da doença e a promoção da saúde.

O programa Hiperdia envolve a realização de ações educativas, trabalhando desde aspectos relacionados ao conhecimento da doença até o autocuidado. Em um trabalho de avaliação do programa no município de Teresina/PI, realizado com 400 pacientes em 10 unidades da Estratégia de Saúde da Família, foi encontrado que $223(55,75 \%)$ usuários desconheciam o nome do remédio que faziam uso, 291 (72,75\%) a desconheciam a dose prescrita, 26 (6,5\%) o intervalo entre as doses e $293(73,25 \%)$ não sabiam até quando iria tomá-los ${ }^{13}$. De acordo com o conhecimento da amostra, os autores consideraram-na não aderente a estratégia ${ }^{13}$. Estudos relevantes no Brasil não consideram a importância dessa falta de conhecimento e adesão para o adoecimento dessa população ${ }^{14,15}$.

Outro trabalho com uma amostra maior, 1.024 usuários das unidades de saúde de Caxias/MA, revelou baixa adesão ao programa pelo número de consultas, com $32,7 \%$ de consulta semestral e $21,0 \%$ anual $^{16}$. Para essa amostra, $44,0 \%$ não apresentam dificuldade em realizar o tratamento, $27 \%$ referem a falta frequente de medicamentos na unidade, $1,9 \%$ a falta de profissionais e 3,2\% residir distante da unidades. Além disso, 71,2\% não participa de nenhuma atividade de educação em saúde, 25,5\% de palestras, $2,1 \%$ de grupos $^{16}$.

Outros autores apontam para o fato de que as ações do Hiperdia se tornam substancialmente mais efetivas quando incluem os familiares ${ }^{17}$. Os apoios emocional, informacional e instrumental originaram-se principalmente dos familiares, caracterizando-se como mais efetivo que os encontros mensais ou consultas isoladas com profissionais ${ }^{17}$. Um estudo randomizado comparando a efetividade da estratégia tradicional de educação através da exposição oral versus uma estratégia combinada com visitas domiciliares e inclusão dos familiares revelou que o grupo que recebia visitas teve uma aderência mais efetiva ao tratamento não-farmacológico e melhora nos fatores de risco comportamentais ${ }^{18}$.

Através de uma investigação de abordagem qualitativa, revelou-se que os enfermeiros reconhecem a importância das ações do programa Hiperdia, mesmo que essa prática remonte a educação em saúde tradicional, com o saber científico em detrimento ao popular ${ }^{19}$.

Ações de promoção da saúde na atenção primária vêm sendo estudadas não apenas no âmbito nacional, havendo evidências de que o seguimento dos pacientes com foco na educação em saúde e na ampliação dos espaços de construção de saber reflete positivamente no controle das doenças crônicas ${ }^{20}$. Um estudo coreano do tipo quase-experimental apontou que o grupo de idosos submetidos a um acompanhamento multiprofissional aliando consultas com estratégias de educação e discussões em

Tabela 2: Análise comparativa entre os resultados dos pacientes acompanhados pelo Programa Hiperdia e o grupo controle. Recife-PE, 2017

\begin{tabular}{|c|c|c|c|c|}
\hline Variáveis & Amostra $(\mathrm{N}=331)$ & $\begin{array}{l}\text { Grupo Hiperdia } \\
\qquad(\mathrm{N}=135)\end{array}$ & $\begin{array}{l}\text { Grupo Controle } \\
\qquad(N=196)\end{array}$ & $p$ \\
\hline Idade (anos) & $53,19 \pm 15,77$ & $49,88 \pm 17,79$ & $55,46 \pm 13,80$ & $0,008^{*}$ \\
\hline$>60$ anos & $117(35,35)$ & $96(71,11)$ & $78(66,67)$ & \multirow{2}{*}{$0,041 \dagger$} \\
\hline$\leq 60$ anos & $214(64,65)$ & $39(28,89)$ & $118(55,14)$ & \\
\hline Masculino & $135(40,79)$ & $43(31,85)$ & $91(46,43)$ & \multirow{2}{*}{$0,008 \dagger$} \\
\hline Feminino & $196(59,21)$ & $92(68,15)$ & $105(53,57)$ & \\
\hline Peso (kg) & $73,43 \pm 15,65$ & $72,07 \pm 18,24$ & $74,36 \pm 13,37$ & $0,014^{*}$ \\
\hline Altura (m) & $1,62 \pm 0,09$ & $1,64 \pm 0,09$ & $1,61 \pm 0,10$ & $0,06^{*}$ \\
\hline $\mathrm{IMC}\left(\mathrm{kg} / \mathrm{m}^{2}\right)$ & $27,8 \pm 4,98$ & $26,66 \pm 5,27$ & $28,58 \pm 4,63$ & $<0,001^{*}$ \\
\hline Peso normal & $92(27,79)$ & $53(39,26)$ & $39(19,9)$ & \multirow{2}{*}{$<0,001 \uparrow$} \\
\hline Sobrepeso e Obesidade & $239(72,21)$ & $82(60,74)$ & $157(80,1)$ & \\
\hline PAS $(\mathrm{mmHg})$ & $136,92 \pm 21,57$ & $128,0 \pm 15,8$ & $143,06 \pm 22,8$ & $<0,001^{\star}$ \\
\hline $\mathrm{PAD}(\mathrm{mmHg})$ & $82,95 \pm 13,76$ & $81,33 \pm 10,49$ & $84,07 \pm 15,55$ & $0,011^{*}$ \\
\hline
\end{tabular}

IMC: índice de massa corporal.

*teste de Mann-Whtiney; †teste de qui-quadrado. 
grupo, ao longo de cinco semanas melhorou a significativamente adesão ao regime terapêutico ${ }^{21}$. Na Austrália, a educação em saúde para o monitoramento domiciliar da pressão arterial foi evidenciado como eficaz para a redução a curto prazo da pressão arterial $^{22}$. Em Viena, 591 pacientes com AVC com histórico de hipertensão arterial foram interrogados sobre conhecimentos e comportamentos de risco e o resultado da investigação revelou que o nível de educação formal estava estritamente relacionado, ou seja, quanto menor a escolaridade, maior a incidência de comportamentos de risco e menor o conhecimento sobre a doença ${ }^{23}$. Esses estudos ressaltam a importância de medidas não-farmacológicas, ao passo que o estudo PURE (Prospective Urban Rural Epidemiological), que avalia 626 comunidades em 20 países, revelou que em países de baixa e média rendas, a população não tem acesso a mais de um anti-hipertensivo e, quando tem disponível, não tem recursos para ter acesso ${ }^{24}$.

Conclui-se que o grupo de pacientes em acompanhamento apresentou resultados melhores que o grupo controle, revelando que as ações desenvolvidas no programa Hiperdia apresentaram resultados favoráveis no manejo da hipertensão e no controle de alguns fatores de risco associados ao desenvolvimento de complicações cardiovasculares. Os resultados avaliados em termos de níveis pressóricos refletem aderência ao tratamento farmacológico e não-farmacológico, promovido pelas ações do programa nas unidades.

É necessário estudar estratégias para aumentar a identificação dessa doença na população em geral e intensificar a abordagem nos fatores de risco, na medida em que esse grupo esteve associado a maiores níveis pressórico e sobrepeso/obesidade. Deve ser considerado que nas comunidades com menor renda e menor acesso à educação e saúde, a cobertura do programa torna-se ainda mais decisiva para melhorar a realidade do adoecimento por doenças crônicas.

Os enfermeiros das unidades de saúde da família devem estar atentos para esse grupo de pacientes e o acompanhamento do controle pressórico tem se mostrado como ferramenta indispensável não só ao manejo terapêutico individual, mas também de forma coletiva pode ser considerado bom indicador da eficiência das ações prestadas. O conhecimento dos níveis pressóricos dos pacientes é primordial, mas deve-se buscar sempre como melhorar a adesão terapêutica ao tratamento, o autocuidado e o vínculo e participação na unidade.

\section{REFERÊNCIAS}

1. Rosini N, Machado MJ, Xavier HT. Estudo da prevalência e multiplicidade de fatores de risco cardiovascular em hipertensos do município de Brusque, SC. Arq Bras Cardiol. 2006;86(3):219-22. http://dx.doi.org/10.1590/S0066-782X2006000300010

2. Rissardo LK, Barreto MS, Oliveira AP, Marcon SS, Carreira L. Influence of hypertension and treatment in the quality of life of elderly. J Nurs UFPE on line. 2012; 6(12):2918-26.

http://dx.doi.org/10.5205/reuol.2265-25464-1-LE.0612201207

3. Carvalho MV, Siqueira LB, Sousa ALL, Jardim PCBV. The Influence of hypertension on quality of life. Arq Bras Cardiol. 2013;100(2):164-74 http://dx.doi.org/10.5935/abc.20130030

4. Benjamin EJ, Blaha MJ, Chiuve SE, Cushman M, Das, SR, Deo R, et al. Heart Disease and Stroke Statistics-2017 update a report from the American Heart Association. Circulation. 2017;135:e1-459. http://dx.doi.org/10.1161/CIR.0000000000000485

5. Malachias MVB, Souza WKSB, Plavnik FL, Rodrigues CIS, Brandão AA, Neves MFT, et al. $7^{\text {a }}$ Diretriz Brasileira de Hipertensão. Arq Bras Cardiol. 2016;107(3supl.3):1-83.

6. Cavalcanti FCR, Gomes ET, Veiga EV, Bezerra SMMS. Profile of health and quality of life assesment of hypertensive patients by the specific instrument minichal-Brazil. J Nurs UFPE. 2013;7(12):6732-40.

http://dx.doi.org/10.5205/reuol.2950-23586-1-ED.0712201302

7. Lunelli RP, Portal VL, Esmério FG, Moraes MA, Souza EN. Adesão medicamentosa e não medicamentosa de pacientes com doença arterial coronariana. Acta Paul Enferm. 2009;22(4):367-73. http://dx.doi.org/10.1590/S0103-21002009000400003
8. Brito DMS, Araujo TL, Galvão MTG, Moreira TMM, Lopes MVO. Qualidade de e percepção da doença entre portadores de hipertensão arterial. Cad Saúde Pública. 2008;24(4):933-40. http://dx.doi.org/10.1590/S0102-311X2008000400025

9. Brasil. Ministério da Saúde. Plano de reorganização da atenção à hipertensão arterial e ao diabetes mellitus. Manual de hipertensão arterial e diabetes mellitus. Brasília: Ministério da Saúde; 2002.

10. Gomes TJO, Silva MVR, Santos AA. Controle da pressão arterial em pacientes atendidos pelo programa Hiperdia em uma Unidade de Saúde da Família. Rev Bras Hipertens. 2010;17(3):132-9.

11. Silveira J, Scherer F, Deitos A, Dal Bosco SM. Fatores associados à hipertensão arterial sistêmica e ao estado nutricional de hipertensos inscritos no programa Hiperdia. Cad Saúde Coletiva. 2013;21(2):129-34.

http://dx.doi.org/10.1590/S1414-462X2013000200005

12. Silva VR, Cade NV, Molina MCB. Risco coronariano e fatores associados em hipertensos de uma unidade de saúde da família. Rev Enferm UERJ. 2012;20(4):439-44.

http://dx.doi.org/10.1590/S1983-14472011000200016

13. Carvalho ALM, Leopoldino RWD, Silva JEG, Cunha CM. Adesão ao tratamento medicamentoso em usuários cadastrados no Programa Hiperdia no município de Teresina (PI). Ciênc Saúde Coletiva. 2012;17(7):1885-92. http://dx.doi.org/10.1590/S1413-81232012000700028

14. Moreira TMM, Gomes EB, Santos JC. Fatores de risco cardiovasculares em adultos jovens com hipertensão arterial e/ou diabetes mellitus. Rev Gaúcha Enferm. 2010;31(4):662-9. http://dx.doi.org/10.1590/S1983-14472010000400008 
15. Lima LM, Schwartz E, Muniz RM, Zillmer JGV, Ludtke I. Perfil dos usuários do Hiperdia de três unidades básicas de saúde do sul do Brasil. Rev Gaúcha Enferm. 2011;32(2):323-9. http://dx.doi.org/10.1590/S1983-14472011000200016

16. Carvalho Filha FSS, Nogueira LT, Viana LMM. Hiperdia: adesão e percepção de usuários acompanhados pela estratégia saúde da família. Rev Rene. 2011;12(n. esp.):930-6.

17. Tavares RS, Silva DMGV. A implicação do apoio social no viver de pessoas com hipertensão arterial. Rev Gaúcha Enferm. 2013;34(3):14-21.

http://dx.doi.org/10.1590/S1983-14472013000300002

18. Ribeiro AG, Ribeiro SMR, Dias CMGC, Ribeiro AQ, Castro FAF, Suárez-Varela MM, et al. Non-pharmacological treatment of hypertension in primary health care: a comparative clinical trial of two education strategies in health and nutrition. BMC Public Health. 2011;11:637.

http://dx.doi.org/10.1186/1471-2458-11-637

19. Menezes Júnior JE, Queiroz JC, Fernandes SCA, Oliveira LC, Coelho SQF. Educação em saúde como estratégia para melhoria da qualidade de vida dos usuários hipertensos. Rev Rene. 2011;12(n.esp.):1045-51.
20. Goldman D, Smith JP. The increasing value of education to healt Soc Sci Med. 2011;72(10):1728-37.

http://dx.doi.org/10.1016/j.socscimed.2011.02.047

21. Lee JK. Evaluation of a medication self-management education program for elders with hypertension living in the community. J Korean Acad Nurs. 2013;43(2):267-75.

http://dx.doi.org/10.4040/jkan.2013.43.2.267

22. Fung CSC, Wong WCW, Wong CKH, Lee A, Lam CLK. Home blood pressure monitoring- a trial on the effect of a structured education program. Aust Fam Physician. 2013;42(4):233-7.

23. Samal D, Greisenegger S, Auff E, Lang W, Lalouschek W. The relation between knowledge about hypertension and education in hospitalized patients with stroke in Vienna. Stroke. 2007;38(4):1304-8

http://dx.doi.org/10.1161/01.STR.0000259733.43470.27

24. Attaei MW, Khatib R, McKee M, Lear S, Dagenais G, Igumbor $\mathrm{EU}$, et al. Availability and affordability of blood pressure-lowering medicines and the effect on blood pressure control in highincome, middle-income, and low-income countries: an analysis of the PURE study data. Lancet Public Health. 2017;2(9):e411-19. http://dx.doi.org/10.1016/S2468-2667(17)30141-X 